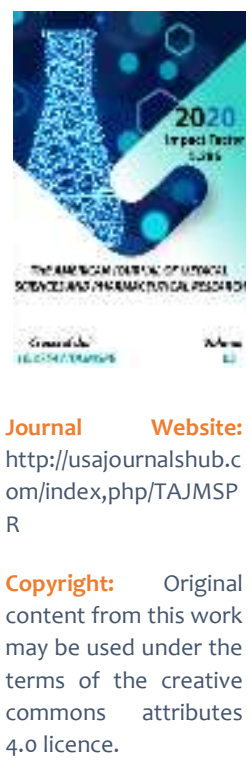

\title{
Efficiency Of Intra-Articular Administration In Early Osteoarthrosis
}

Islamova K. A .

Samarkand State Medical Institute Of The Ministry Of Health Of The Republic Of Uzbekistan

Khasanov F. Sh

Samarkand State Medical Institute Of The Ministry Of Health Of The Republic Of Uzbekistan

Toirov E. S.

Samarkand State Medical Institute Of The Ministry Of Health Of The Republic Of Uzbekistan

\section{ABSTRACT}

The purpose of this study was to investigate the function of knee joints in patients with early osteoarthritis (OA, according to the criteria of the American rheumatology Association 2010) stage IIII using visual analogue scale (VAS) for pain intensity and treatment outcomes, Lequesne index, expert indicators, samples of 4 meter walk to assess the effectiveness of intra-articular injection Hyaluronic Chondro. 80 patients aged 28 to 55 years were examined. The treatment regimen included recommendations from the European rheumatology League (EULAR, 2016) and Russia (Nasonov E. L., 2017). The results of therapy were evaluated in two groups. The first group included 43 patients who received Hyaluronic Chondro in the form of intra-articular injections, the second-37 patients who received chondroprotectors inside or in the form of intramuscular injections. Conclusion. Intraarticular administration of the drug Chondrogard has a positive clinical effect in early stage II-III OA with moderate impairment of knee joint function. The course of treatment should last at least 2 months

\section{KEYWORDS}

Osteoarthritis, the Hyaluronic Chondro, the Lequesne index, the expert indicators, test 4 meter walk.

\section{INTRODUCTION}

A common form of $\mathrm{OA}$ that contributes to temporary or permanent disability is a lesion of the knee joint-gonarthrosis (Kornilov N. N., 2013; Kashevarova N. G., Alekseeva L. I., 2014;
Kornilov N. N., Denisov A. A., 2017; Lisitsyna E. M., Lisitsyn M. P., Zaremuk a.m., 2016). Damage to the subchondral bone with subsequent violation of bone remodeling, formation of 
osteophytes, subchondral sclerosis (Mustafin R. N., Khusnutdinova E. K 2015; Kabalyk M. A., 2016; Atukorala I., Kwoh C. K., Guermazi A., et al., 2016) in gonarthrosis is reflected in a decrease in the functional ability of both the knee joint or limb itself and the musculoskeletal system as a whole. Despite a sufficient number of diagnostic approaches, including radiography, ultrasound (ultrasound), computed tomography (CT) and magnetic resonance imaging (MRI), the slow development of the pathological process and the absence of severe pain at an early stage is usually the main reason for the late diagnosis of OA (Kornilov N. N., 2013; Makarova M. V. et al., 2017; Filatova E. S., Turovskaya E. F., Alekseeva L. I., 2018).

Currently, it is important to identify early clinical signs and causes of the disease, determine the degree of joint damage and early start of therapeutic measures to restore the function of damaged joints (Lisitsyna E. M., Lisitsyn M. P., Zaremuk A.M., 2016; Makarova M. V. etc., 2017; Paula A. Hernandez et al., 2020). This is justified by the fact that $O A$ at late stages becomes a problem only for the practice of endoprosthetics or palliative treatment (Kashevarova N. G., Alekseeva L. I., 2014; Lisitsyna E. M., Lisitsyn M. P., Zaremuk A.M., 2016; Kornilov N. N., Denisov A. A., 2017; Karateev A. E., 2019).

According to the criteria of the College of Reumatology ( $A C R, 2010)$, diagnostic signs of early OA are (sensitivity $91 \%$, specificity $86 \%$ ) episodes of pain in the knee joint (at least 10 days in the last 2 years), the presence of stiffness, crepitation and structural changes, i.e. osteophytes and narrowing of the joint gap (Kellgren II on standard radiographs) (Kelli D. Allen, Yvonne M. Golightly, 2015). Luyten F. P., Denti M., Filardo G., Kon E., Engebretsen L., (2012)along with osteophytes, the criteria for early $\mathrm{OA}$ also recommend including signs of cartilage degeneration (damage to ligaments, miniscus, subchondral bone) detected by MRI.

Since the main pathogenesis of $O A$ is dystrophic changes in articular cartilage and loss of proteoglycans, a number of drugs are proposed for the basic (main) treatment of the disease, the mechanism of action of which is aimed at compensating for the loss of cartilage and stimulating the synthesis of hyaluronic acid and chondroitin sulfate - natural components of cartilage tissue. Hyaluronic acid is also found in synovial fluid ,which "lubricates" the articular surfaces of bones, reducing their friction (Anikin S. G., 2017; Anikin S. G. et al., 2017; Petukhov A. I., Kornilov N. N., Kulyaba T. A., 2018; Nesterenko V. A., 2019; Mirakhmedova H. T., Dadabaeva N. A., Ramazonova N. A., 2019).

The drug Hyaluronic Chondro is a drug for intraarticular administration. $3 \mathrm{ml}$ of the solution contains $60 \mathrm{mg}$ of sodium hyaluronate and 90 $\mathrm{mg}$ of chondroitin sulfate. The drug is indicated for the treatment of pain and stiffness caused by degenerative or traumatic pathologies of the knee and other synovial joints (hip, ankle, shoulder, elbow, wrist, fingers, temporomandibular joint, arcuate joints). Chondro hyaluronic acid is also used to relieve pain after arthroscopy.

Based on the above, the aim of our work was to study the dynamics of joint syndrome in patients with gonarthrosis mainly I-III x-ray stages using the index, Lequesne, expert indicators and a 4-meter walk test to assess the effectiveness of treatment with Hyaluronic Chondro.

MATERIALS AND METHODS OF RESEARCH 
The work was carried out in the Department of rheumatology of the clinic No. 1 of the Samarkand state medical Institute. The results of the study are based on data from the examination and treatment of 80 patients ( 57 women, $71.2 \%$; 23 men, $28.8 \%$ ) with OA of the knee joint. The diagnosis of OA was established in accordance with the Altman (1991) criteria, taking into account the criteria proposed by The Institute of rheumatology of the Russian Academy of medical Sciences (Nasonov E. L., 2017).

The degree of pain intensity was determined using a visual analog scale (VAS). Measurement scales were used to assess joint function and OA severity, including the Lequesne index, expert criteria, and a 4-meter walk test. Radiography and ultrasound examination of the joints were performed using imaging diagnostic methods, and magnetic resonance imaging of the joints was performed as needed.

X-ray signs of OA were detected in 57 (71.3\%) patients. According to the criteria of Kellgren and Lawrence (1957), 20 (25.0\%) patients had stage I (non - specific expression of rengenological signs and poorly developed osteoclerosis), 25 (31.3\%) patients had stage II (minimal changes - joint narrowing, primary osteophytes) and 12 (15.0\%) patients had stage III (moderate changes - joint narrowing, increased osteophytes) of the disease. In 23 (28.7\%) patients, x-ray examination did not show any changes.

According to medical documents, 15 (18.8\%) patients had a joint dysfunction (NFS): 9 (11.3\%) - first degree (professional ability is preserved), 6 (7.5\%) patients - second degree (professional disability is lost). Functional activity of the knee joints was preserved in 65 (81.2\%) patients. There were also no patients with third-degree NSF and stage IV x-ray disease among OA patients.

Treatment of patients with OA was carried out in accordance with the recommendations of the European rheumatology League (EULAR, 2016) and Russia (Nasonov E. L., 2015; 2017), providing for pain reduction (non-steroidal anti-inflammatory drugs, NSAIDs), stopping degeneration of articular cartilage (chondroprotectors, "basic therapy") and improving the functional activity of the joints (physiotherapy, physical therapy procedures).

NSAIDs (Meloxicam at a dose of $15 \mathrm{mg} /$ day, Etodolac $400 \mathrm{mg} /$ day. Nimesulide $200 \mathrm{mg} /$ day) were recommended individually to each patient (mainly for oral administration and intramuscular injections), chondroprotecors (chondriatin sulfate, chondriatin and glycosamine sulfate) were applied orally and in the form of intra-articular and intramuscular injections. To improve microcirculation, the antiplatelet Curantil was recommended (at a dose of $0.075 \mathrm{mg} /$ day), in order to reduce bone resorptive processes and osteoporosis, calcium preparations (Calciques, Vitamin D3 calcimed, Forcal).

43 patients received Chondro Hyaluronic acid by injection into the knee joint. The procedures were performed once a week. The course of intra-articular injections consisted of 2 to 4 injections (depending on the degree of improvement in the signs of joint syndrome). Before the drug was administered, an ultrasound examination was performed to determine the injection site. The tolerability of the procedures was good, only during the injection, patients felt some tingling when the skin was pierced with a needle and the medication was administered.

The results of General treatment were evaluated in two groups. The first group 
included 43 patients who used Hyaluronic Chondro in the form of intra-articular injections, the second group included 37 patients who received chondroprotectors inside or in the form of intramuscular injections. Depending on the gender and age of patients, the duration of the disease, the comparative groups did not have sharp differences.

Monitoring of the effectiveness of treatment was carried out in stages: after discharge from the hospital (end of 2 weeks), after 4 and 8 weeks. Statistical data processing was performed using the computer program "Statistica 12.0".

\section{RESULTS}

In our observations, starting from the end of the first week, patients began to notice a decrease in joint pain. The positive dynamics began with the disappearance of pain in the knee joint at rest. By the end of the second week, there was a decrease and disappearance of pain when walking on a straight road, when descending and climbing stairs, when bending and extending the knee joint. By the end of the month, there was a significant decrease in VAS indicators both at rest and when moving.

After 8 weeks from the beginning of treatment in patients of the main (first) group, complete disappearance of pain syndrome was observed much more often - in $90.6 \%$ of patients at rest, in $72.9 \%$ of patients when moving, and in the control (second) group - in 81.8 and $62.6 \%$ of patients, respectively. Patients were particularly noted for such positive results as a reduction or disappearance of pain when performing daily household chores (cooking, cleaning rooms, washing, caring for children, etc.), personal hygiene procedures, and reducing difficulties when using public or private transport.
Due to the lack of validated parameters that reflect the state of the joints in patients with early OA (Rustamova U. M., Umarova G. Sh., 2015; Nasonov E. L., 2015; 2017), to study the features of the clinical course of the disease and to assess the response to therapy, we used indicators of the Lequesne index, expert signs, and 4-meter walking tests developed to assess the functional status of patients.

When assessing the severity of lower limb function disorders, an integral indicator was used, which is calculated as the arithmetic mean of the value of 6 expert signs in \% (Nasonov E. L., 2015; 2017). Before treatment, according to the first expert indicator (determination of the ability to move), $42.5 \%$ of patients (41.9\% in the first group, $43.2 \%$ in the second group) had pronounced (21-40\%), 7.5\% of patients (6.9\% in the first group, $8.1 \%$ in the second group) had pronounced (41-60\%) lameness. According to the second feature (additional support), $11.3 \%$ of patients ( $9.3 \%$ in the first group, $14.7 \%$ in the second group) needed additional support (21-40\%, a walking stick). $12.5 \%$ of patients (11.6\% in the first $13.5 \%$ of the second group) had a limitation in performing household functions and selfservice (41-60\%, third and fourth peer basis), $10 \%$ of patients ( $9,3 \%$ in the first, $10,8 \%$-in the second group) - with the use of public transport and job performance (41-60\%, fifth and sixth expert feature).

In General, the arithmetic mean value of 6 expert algorithms in $38.8 \%$ (34.9\% in the first group, $43.3 \%$ in the second group) of patients exceeded $20 \%$ (on average $34.4 \pm 5.6 \%$ ) and corresponded to moderate violations. The results of the study of expert signs to a certain extent coincided with the measurement data of the Lequesne index, where in $27.5 \%$ (25.6\%-in the first, $29.7 \%$-in the second group) cases, moderate OA was detected, in $12.5 \%$ (11.6\% - in 
the first, $13.5 \%$-in the second group) cases severe.

A reduction in walking speed based on the height and gender of patients was measured using the method of Fried et al. (2001) with guidance from Andrews J. S., Trupin L., Yelin E. H., Hough C. L., Covinsky K. E., Katz P. P. (2017). The walking speed of 4 meters was taken into account. For men shorter than or equal to 1.73 meters speed reduction was considered the time to travel this distance in 7 seconds or more ( $\geq 7$ ), a growth of over $1.73 \mathrm{~m}$ in 6 seconds or more $(\geq 6)$. For women, a slow 4-meter walking speed was defined as: $\geq 7$ seconds for height $\leq 1.59$ meters and $\geq 6$ seconds for height $>1.59$ meters.

Before treatment, a decrease in walking speed with a 4-meter test was observed in 13 (16.3\%) patients: $9(11.3 \%)$ women, 4 (5.0\%) men. In the first group, a decrease in walking speed was observed in $6(14.0 \%$ ) patients (4 women, 9.3\%, 2 men, $4.7 \%)$, in the second group - in 7 (18.9\%) patients ( 5 women, $13.5 \%$, 2 men, 5.4\%).

The treatment also contributed to the improvement of the above-mentioned functional measurements. By the end of 2 months from the start of therapy, there was a decrease in the average value of expert signs from $34.4 \pm 5.6 \%$ to $12.5 \pm 3.6 \%(P<0.02)$, and in only $15 \%$ (11.6\% - in the first group, $18.9 \%$ - in the second group) of patients, the total indicator exceeded $20 \%$.

By this time, the Lequesne index had improved. So, by the end of 2 months, the number of patients with mild OA (1-4 points) increased by 1.4 times (from $60.0 \%$ to $85.0 \%$ ), and the number of patients with moderate (5-7 points) and severe OA (8-10 points) decreased by 2.7 times (from $40.0 \%$ to $15.0 \%$ ). Among the first group of patients, the number of patients with moderate OA decreased from $25.6 \%$ to $11.6 \%$, and the number of patients with severe OA decreased from $11.6 \%$ to $6.9 \%$. The effectiveness of treatment of patients in the control group (the second group) was also good, but the degree of improvement in the Lequesne index was inferior to the results of the main group. At the last examination in the control group, patients with moderate OA were $16.2 \%$, severe OA-8.1\%.

There was also a positive dynamics in the 4meter walking test: in the first group, only 2 (4.7\%) patients had a decrease in walking speed, while in the second group, $3(8.1 \%)$ patients had a decrease in walking speed.

The overall assessment of treatment effectiveness was carried out jointly with patients based on the results of VAS. The initial assessment of the General condition was indicated by $10 \mathrm{~cm}$ (before treatment). A good result was considered to be a decrease in VAS by $5-6 \mathrm{~cm}$, satisfactory-by $3-4 \mathrm{~cm}$, not satisfactory (no changes) - by $1-2 \mathrm{~cm}$.

\section{CONCLUSION}

Complex treatment with the inclusion of intraarticular injections of the drug Hyaluronic Chondro has a fairly high therapeutic effectiveness in patients with early gonarthrosis mainly of stages II-III. When treated with the drug, the effect occurs in 1-2 weeks. To clarify the benefits of this method of treatment, it is necessary to conduct more large-scale, controlled studies that allow us to observe long-term treatment results $(\geq 6$ months).

\section{REFERENCES}

1. Alekseeva L. I., taskina E. A., Kashevarova N. G. Osteoarthritis: epidemiology, classification, risk factors and progression, clinic, diagnosis, treatment. // Modern rheumatology. 2019. No. 13 (2). pp. 9-21. 
2. Anikin S. G. the Use of high-molecular hyaluronic acid preparations in the treatment of osteoarthritis. // Modern rheumatology. 2017. No. 11 (1). pp. 62-65.

3. Anikin S. G., Kashevarova N. G., Korotkova T. A., etc. Evaluation of the efficacy, tolerability and safety of intra-articular administration of hyaluronic acid in patients with knee osteoarthritis. // Modern rheumatology. 2017. No. 11 (3). pp. 58-63.

4. Belyaeva I. B., Lila a.m., Mazurov V. I., Raimuev K. V. Algorithm of pharmacological treatment of knee osteoarthritis (recommendations of ESCEO-2016). // Rheumatology, traumatology and orthopedics. 2017. No. 2. pp. 8-13.

5. Benevolenskaya L. I., Brzezovsky M. M. Epidemiology of rheumatic diseases. Moscow, 2012. 237 p.

6. Denisov L. N., Platova A. I., Menshikova I. V., Lila a.m. Osteoarthritis-aspects of pharmacotherapy. I/ Modern rheumatology. 2018. No. 2. pp. 97-102.

7. Diagnosis and treatment of certain forms of rheumatic diseases from the position of evidence-based medicine. Educational and methodological guide. S. K. Adzhigaytkanova. M., GBOU VPO RNIMU named after N. I. Pirogov, 2013. 52 p.

8. Kabalyk M. A. Textural characteristics of subchondral bone in osteoarthritis. // Kazan medical journal. 2016; Vol. 97. no. 4. pp. 518-523.

9. Karataev A. E., Lila a.m. Osteoarthritis: modern clinical concept and some promising therapeutic approaches. // Scientific and practical rheumatology. 2018. No. 56 (1). pp. 70-81.

10. karateev A. E. the Problem of musculoskeletal pain: what is interesting for a practicing doctor? Data from a survey of 846 Russian doctors. // Modern rheumatology. 2019. No. 13 (2). pp. 126-129. 